\title{
Geometry in Partial Differential Equations
}


This page is intentionally left blank 

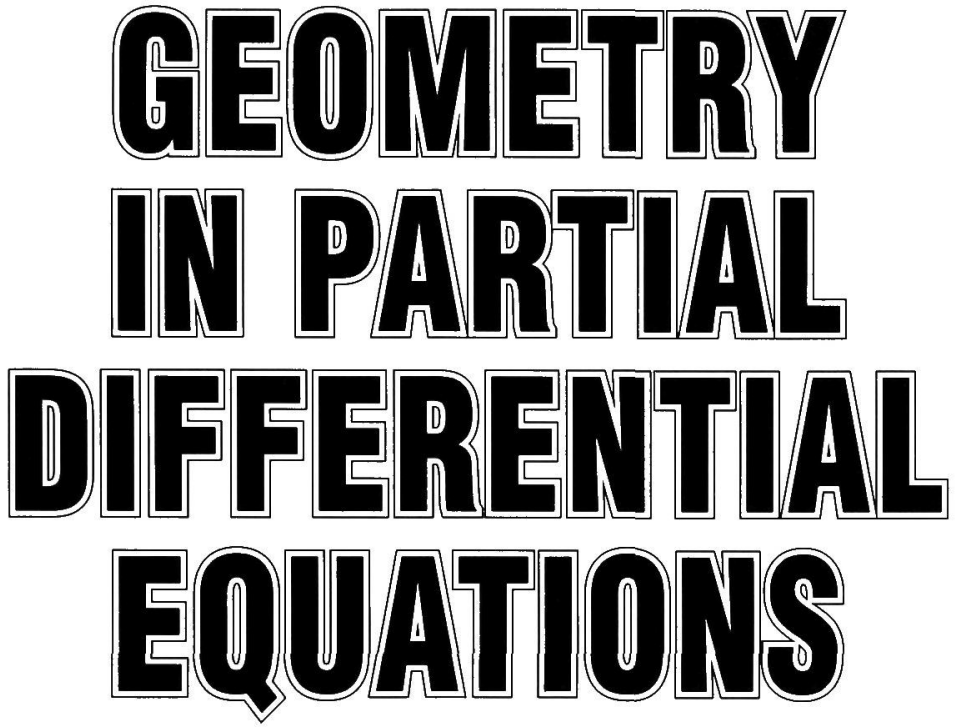

\section{A. Pràstaro \\ Rome, Italy \\ Th. M. Rassias \\ Athens, Greece}


Published by

World Scientific Publishing Co. Pte. Ltd.

P O Box 128, Farrer Road, Singapore 9128

USA office: Suite 1B, 1060 Main Street, River Edge, NJ 07661

UK office: 73 Lynton Mead, Totteridge, London N20 8DH

\section{GEOMETRY IN PARTIAL DIFFERENTIAL EQUATIONS}

Copyright (c) 1994 by World Scientific Publishing Co. Pte. Ltd.

All rights reserved. This book, or parts thereof, may not be reproduced in any form or by any means, electronic or mechanical, including photocopying, recording or any information storage and retrieval system now known or to be invented, without written permission from the Publisher.

For photocopying of material in this volume, please pay a copying fee through the Copyright Clearance Center, Inc., 27 Congress Street, Salem, MA 01970, USA.

ISBN $981-02-1407-3$

Printed in Singapore by JBW Printers \& Binders Pte. Ltd. 


\section{PREFACE}

Geometric tools have been of particular importance to the understanding of the structure of Partial Differential Equations (PDEs). This has been shown in the pioneering works of eminent mathematicians such as L. Bianchi (1856-1928), C. Carathéodory (1873-1950), E. Cartan (18691951), L. Euler (1707-1783), C. F. Gauss (1777-1855), T. Levi-Cirita (1873-1941), S. Lie (1842-1899), E. Noether (1882-1935), H. Poincaré (1854-1912), G. Ricci-Curbastro (1853-1925), B. Riemann (1826-1866), H. Weyl (1885-1955), and, during recent times, in the works of C. Ehresmann, H. Goldschmidt, M. Kuranishi, B. Malgrange, D. Spencer, S. Steinberg, A. M. Vinogradov, among others. Differential Geometry and Algebraic Topology play a fundamental role in the development of a geometrical framework for the understanding of PDEs. In fact the first important step that has been taken in this direction has been the introduction of manifolds with some particular structure, which represent the natural environment for a PDE. This consists of the jet derivative spaces, first strictly formalized by C. Ehresmann. This way, PDEs can be regarded as submanifolds of these types of spaces and thus the machinery of Differential (Algebraic and Topological) Geometry is naturally introduced. In this context the Analysis of the space of solutions can be regarded as a sub-product of a more general geometric theory. In fact, what is important in the characterization of PDEs is not so much their space of solutions but rather the manifold that defines them.

The big advantage in the study of PDEs from the geometric point of view is the possibility of investigating the general behavior of their structure, without considering the set of solutions and without going into many particular cases. Moreover, when one uses for example modern Cartan theory one can obtain general results about the characterization of singular solutions of PDEs. This aspect is particularly interesting also from the applications point of view, where singular solutions can play a significant role in order to explain several fundamental phenomena.

It is important also to underline the current developments in the geometric approach. A few such approaches include: general methods to obtain conservation laws for PDEs, structural investigation for the understanding of the meaning of quantum geometry in PDEs, extensions to super PDEs 
(formulated in the category of supermanifolds) of the geometrical methods introduced for PDEs. The harmonic theory proved to be very important after the appearance of the Atiyah-Singer index theorem, which provides a link between geometry and topology.

The Yang-Mills equations are of interest to both physicists and mathematicians. M. Atiyah and R. Bott found applications of the Yang-Mills theory in the study of vector bundles over Riemann surfaces. After S.-T. Yau's solution of the Calabi conjecture several surprising results followed in Geometry and Topology. These developments include the solution of the positive mass conjecture and other applications of minimal surfaces, S. Donaldson's construction of four manifold invariants using the moduli spaces of connections, among other results. The most important quasilinear equation in Geometry is the minimal surface equation. Minimal surfaces have, over a long period of time, been a powerful tool in understanding the topology and the geometry of manifolds. Minimal surfaces also play a very important role in the study of the curvature of a manifold (works of J. Sacks and K. Uhlenbeck, R. Schoen and S.-T. Yau are very representative in this direction). The first problem in minimal surface theory is the Plateau problem. The works of L. Ljusternik and L. Schnirelman and of Marston Morse provide two important approaches towards the foundation of global analysis. In the 60's Morse theory was generalized by R. Palais and S. Smale to infinite-dimensional Hilbert manifolds. The Morse-Palais-Smale theory has proved to be useful (and usable) in the study of PDEs.

This volume emphasizes the interdisciplinary interaction in problems involving Geometry and Partial Differential Equations. It provides an attempt to follow certain threads that interconnect various approaches in the applications of geometry and their influence to PDEs.

We wish to express our wholehearted thanks to the scientists who contributed their works in this book and, last but not least, the World Scientific Publishing Company for their excellent cooperation. 


\section{CONTENTS}

Preface

Some Applications of the Coarea Formula to Partial

Differential Equations

F. Bethuel, J.-M. Ghidaglia

Large Solutions for the Equation of Surfaces of Prescribed

Mean Curvature

F. Bethuel, O. Rey

Optical Hamiltonian Functions

M. Bialy, L. Polterovich

On the Geometry of the Hodge-de Rham Laplace Operators

M. Craioveanu, M. Puta, Th. M. Rassias

Minimal Surfaces in Economic Theory

J. Donato

The Morimoto Problem

B. Doubrov, A. Kushner

Asymptotic Expansions in Spectral Geometry

P. B. Gilkey

Deformations and Recursion Operators for Evolution Equations

I. S. Krasil'shchik, P. H. M. Kersten

Geometric Hamiltonian Forms for the Kadomtsev-Petviashvili and Zabolotskaya-Khokhlov Equations

B. A. Kupershmidt

Classification of Mixed Type Monge-Ampere Equations 
Non-Holonomic Filtration: Algebraic and Geometric Aspects of Non-Integrability

V. Lychagin, V. Rubtsov

Spencer Cohomologies

V. Lychagin, L. Zilbergleit

Hawking's Radiation via Fourier Integral Operators

P. E. Parker

Geometry of Super PDE's

A. Pràstaro

On a Geometric Approach to an Equation of J. d'Alembert

A. Pràstaro, Th. M. Rassias

Geometric Prequantization of the Einstein's Vacuum Field Equations

M. Puta

On Differential Equations and Cartan's Projective Connections $Y$. R. Romanovsky

Smooth Marginal Analysis of Bifurcation of Extremals Yu I. Sapronov

On the Schrödinger Equation for an $N$-Electron Atom C. S. Sharma

Higher Symmetries and Conservation Laws of Euler-Darboux Equations

V. E. Shemarulin

Strings and Membranes

K. S. Stelle 
Methods for Solving Two-Dimensional Nonstationary MHD

Equations at Small Alfven-Mach Numbers

V. S. Titov

Author Index 